Relations industrielles

Industrial Relations

\title{
Bernoux, Philippe, La sociologie des entreprises
}

\section{Denis Harrisson}

Volume 51, numéro 1, 1996

URI : https://id.erudit.org/iderudit/051087ar

DOI : https://doi.org/10.7202/051087ar

Aller au sommaire du numéro

\section{Éditeur(s)}

Département des relations industrielles de l'Université Laval

\section{ISSN}

0034-379X (imprimé)

1703-8138 (numérique)

Découvrir la revue

Citer ce compte rendu

Harrisson, D. (1996). Compte rendu de [Bernoux, Philippe, La sociologie des entreprises]. Relations industrielles / Industrial Relations, 51(1), 220-222.

https://doi.org/10.7202/051087ar

Tous droits réservés (C) Département des relations industrielles de l'Université Laval, 1996
Ce document est protégé par la loi sur le droit d'auteur. L'utilisation des services d'Érudit (y compris la reproduction) est assujettie à sa politique d'utilisation que vous pouvez consulter en ligne.

https://apropos.erudit.org/fr/usagers/politique-dutilisation/ 
employed are detailed, such as rotating strikes between different departments in a plant. And the margins of collective organization, which did not extend to large areas of the country, to smaller firms or to white collar or technical workers, are traced.

An additional point refers to the structuring of the volume. The book is organized as a continuing narrative, what Franzosi refers to as 'my story' (p. 348). With the exception of the opening and closing chapters, each chapter takes up what remains unexplained from its antecedent. This allows a single theme to be developed incrementally at length, which is a commendable aim. But it is difficult to read the chapters out-of-sequence, or as self-contained pieces. This limits the book's usefulness for teaching purposes. It would also have been beneficial for the explanatory model, which is introduced only in the final chapter of the book, to have been described at the outset. In this way, the model might have been compared to the competing theories which are variously tested.

It is a theoretically challenging work. Franzosi advances an explanatory model which is founded upon the French Régulation and the American social structure of accumulation schools of social thought. It is a neo-Marxian account of the reciprocity between social conflict and the institutional mechanisms that regulate it. This is the basis of a profound explanation. But it is not fully satisfying. The foundational elements of the model accumulation, class conflict, class structure and economic/political organization need to be expanded. As a result, the model, as set out in the text, is insufficiently detailed to provide the clinching argument that is required. There is great potential to develop further this thesis.

MARTIN WRIGHT The University of Warwick

\section{La sociologie des entreprises}

par Philippe BERNOUX, Paris, Éditions du Seuil, 396 p., ISBN 2-02-023632-X.

Depuis la publication en 1985 du volume Sociologie de l'organisation, le sociologue français Philippe Bernoux a permis à plusieurs lecteurs, étudiants, professeurs et praticiens de s'initier à la sociologie des organisations par cet ouvrage didactique et érudit sur un sujet fort complexe et mouvant, l'organisation du $\mathrm{XX}^{\mathrm{e}}$ siècle. Cette fois, l'auteur publie un volume fort relevé sur la sociologie des entreprises qui complète en même temps qu'il marque une rupture avec les organisations comme objet sociologique. En effet, nous dira l'auteur, la sociologie des entreprises prend le relais de la sociologie du travail et de celle des organisations. Mais elle rompt avec celles-ci parce que l'objet en est renouvelé, le champ d'étude se distinguant des objets sociologiques précédents.

Mais il ne suffit pas de le dire, encore faut-il que l'objet soit construit de façon convaincante. D'autant plus que la notion de sociologie de l'entreprise a cours essentiellement dans la sociologie française. Ailleurs, principalement dans le monde anglo-saxon, la sociologie des organisations tient encore lieu de domaine d'étude et d'analyse des lieux de production et de coordination d'activités humaines, il sera question d'organisation " post-moderne", pour bien distinguer les transformations en cours, qui se démarque de l'organisation bureaucratique. Outre cette distinction dénominative, l'objet est-il le même? Après tout il existe bien une "sociologie industrielle", notamment aux États-Unis, qui n'a jamais eu d'écho en France. Philippe Bernoux expliquera que l'entreprise est un objet sociologique en rupture avec l'organisation depuis le début de la décennie 80 où l'on assista d'abord à son " réenchantement " suite à la crise économique. Puis les travaux précurseurs de Renaud 
Sainsaulieu et de Denis Segrestin sur la dynamique culturelle de l'entreprise font de celle-ci non pas uniquement un lieu de production économique mais aussi de création des liens sociaux dans un espace lié institutionnellement à la société. En construisant l'objet, l'auteur se démarque de l'approche culturaliste, il cherche d'abord à enrichir la sociologie des entreprises d'une approche plus générale.

L'essentiel de la thèse de Bernoux repose sur deux arguments. D'abord, la sociologie de l'entreprise se distingue de la sociologie de l'organisation par le regard porté sur l'objet. L'entreprise n'est pas que le lieu de coordination des actions qui définit les organisations, mais elle est le lieu de création d'un lien social particulier dans lequel se crée des accords et des identités. L'entreprise n'est pas statique, elle connait des changements radicaux qui en font un lieu d'apprentissage de la coopération. Ensuite, les développements théoriques récents fournissent aux analystes de nouveaux outils pour appréhender la réalité des entreprises qui vont bien " au-delà de l'organisation ". Une bonne partie du volume est consacrée à la présentation de ces nouvelles théories empruntées parfois à d'autres domaines de la sociologie et de l'économie.

Le fondement du projet repose sur le rejet de certaines formulations théoriques classiques de l'entreprise comme étant incomplètes : réductionisme aux individus qui composent l'entreprise dans une forme de psychologisme des acteurs; réductionisme également dans le modèle rationnel fondé sur le marché et la bureaucratie.

L'entreprise pourrait-elle alors n'être qu'un construit par les acteurs? Avec un certain mérite, quatre thèses appuient la réponse de l'auteur : l'analyse stratégique et le pouvoir de Michel Crozier que l'auteur ne rejette pas mais qu'il trouve incomplète à l'analyse de l'ensemble des enjeux sociaux dans l'entreprise ; la construction des règles de Jean-Daniel Rey- naud, une approche qui s'est enrichie d'un certain nombre de travaux empiriques importants ; l'étude de l'innovation par la traduction de Michel Callon, Bruno Latour et Madeleine Akrich, une théorie innovatrice en science et en technologie mais qui a peu servi à ce jour à l'analyse sociologique des entreprises ; l'économie des conventions de Robert Salais et André Orléan, une école de pensée économique prometteuse qui n'est qu'au début de son programme de recherche. Bernoux présente ces approches théoriques dans lesquelles les règles n'ont de sens que celui que leur donnent les acteurs qui les construisent. Les liens sociaux se forment par la confiance, thème central qui resurgit, et par la lutte identitaire des acteurs comme processus de constitution des conventions et des accords. Bernoux ne cherche pas à raccorder ces thèses, chaque ensemble théorique montre une facette de la réalité. Selon l'auteur, une théorie ne peut à elle seule tenir compte de situations diverses qu'au prix de lacunes dommageables à l'analyse.

Après avoir exposé et analysé de façon brillante et claire certains concepts propices à l'étude des relations sociales dans l'entreprise, l'auteur développe ce qu'il appelle sa posture mêthodologique où il introduit la notion pluraliste de logique d'action afin de rompre avec les déterminismes. La notion de logique d'action serait plus souple que la logique d'acteur en ce qu'elle permettrait de mieux saisir les enjeux en évolution. En effet, la logique d'acteurs repose sur le pouvoir que détiennent ces derniers représentés dans l'analyse comme étant statiques. À l'opposé, la dynamique évolutive de l'action tient compte du passé des acteurs, de leurs interactions, de leur situation qui structurent les jeux de pouvoir et les compromis. En accord avec les prémisses théoriques selon lesquelles la diversité situationnelle de la réalité ne se comprend qu'au prix du pluralisme théorique, la posture méthodologique de Bernoux y fait écho en renonçant aux 
explications universelles : "Toute situation d'entreprise relève d'un niveau d'explication chaque fois différent"(p. 244). L'argumentation est complétée par des exemples et la dernière partie de l'ourrage est consacrée à l'étude empirique de quatre cas qui illustrent des situations d'identité et de culture; de traduction; de conventions ; de construction des règles. L'auteur préfère une position où il fait face à des faits sociaux qu'il doit interpréter en puisant dans des théories sans a priori.

Voilà un ouvrage qui innove sur plusieurs plans: (a) l'entreprise comme un objet autonome qui se détache de l'analyse des organisations; (b) les préceptes théoriques dont certains concepts n'ont pas été couramment utilisés à l'analyse des jeux d'acteurs dans l'entreprise, notamment la traduction utilisée essentiellement en sociologie de la science et de la technologie, les conventions qui servent d'amorce à un nouveau programme de recherche; la confiance qui malgré sa résurgence reste un concept vague ; (c) la posture méthodologique qui emprunte à Feyerabend le "tout est bon " et cherche à tenir compte d'une pluralité de situations évolutives. Cela semble excessif mais seule l'épreuve du temps saura nous indiquer si ces innovations traverseront les bouleversements que connaît l'entreprise comme objet d'analyse que ces concepts aideront à faire comprendre et à expliquer.

Le débat sur l'existence d'une sociologie de l'entreprise ne s'achèvera certainement pas avec la publication de ce volume. Au contraire, il est lancé à supposer que les sociologues en provenance d'autres horizons culturels et théoriques prendront le relais et sauront y intégrer d'autres développements sur les organisations post-modernes et l'entreprise. Par exemple, les ouvrages de Stewart R. Clegg, Modern Organizations: Organization Studies in the Postmodern World (Sage Publications, London, 1990) et de John Hassard et Martin Parker, Postmodernism and Organizations (Sage Publications, London, 1993) rompent également avec les positions conceptuelles et méthodologiques de la sociologie classique des organisations. Bernoux présente essentiellement les dernières positions théoriques développées en France, ce qui témoigne de la vitalité de la sociologie française. Ailleurs, le débat porte moins sur la constitution d'un nouvel objet que sur la rupture d'avec la conception classique des organisations. Il n'est pas question de désigner l'objet autrement que par le terme " organisation " qui comprend également des lieux de coordination d'activités non productives tels les syndicats, les partis politiques, les Églises, les organismes à but non lucratif, etc.

En somme, voilà un ouvrage qui constitue une bonne synthèse des innovations conceptuelles sur l'entreprise et qui sont clairement exposées par Philippe Bemoux. Le livre ne fait pas que le point sur le sujet, il propose des pistes de recherche et renouvelle le champ d'analyse avec de nouveaux outils conceptuels et des applications empiriques. La lecture est stimulante et je la recommande à tout praticien, étudiant et professeur intéressés par le sujet.

DENIS HARRISSON

Université du Québec à Hull

\section{La vie dans les organisations: des indicateurs de succès}

par Roch LAFLAMME, Sainte-Foy, Presses de l'Université du Québec, 1994, 59 p., ISBN 2-7605-0782-3.

La monographie de 60 pages de Roch Laflamme présente un bon résumé de quatre concepts de base importants en comportement organisationnel. Ce sont les concepts de satisfaction au travail, de climat organisationnel, de qualité de vie au travail et de culture organisationnelle. L'auteur conclut son travail par une des- 